\title{
Discrete Multi-Tone (DMT) Modulation Based Multiple Access Resource Allocation for Visible Light Communication
}

\author{
Dasari Subba Rao, N.S.Maruti Sarma
}

\begin{abstract}
Paper Discrete multi-tone (DMT) adjustment is accepted to be a accomplished single-transmitter arrangement for apparent ablaze correspondence. Be that as it may, the appliance of this action in a abundant transmitter action requires acute subcarrier and ability administration agreement so as to corruption the best accommodation of spatial altered transmitter different variety. Spatial reclaim of the subcarriers aural the afterimage of impedance and ability requirements expands the capability of abundant access (MA) DMT correspondence. In this paper, we adduce a adding that oversees obstruction accountable subcarrier reclaim amid assorted transmitters and ability redistribution amid assorted subcarriers in a heuristic way. The adding reenactment demonstrates an accessory in the accustomed bitrate as assorted and a acceptable DMT technique. Besides, the activity of the proposed MA-DMT artifice increments with the abundance of clients
\end{abstract}

Index Terms: Admission control, QoS, Optical wireless communication, WSN.

\section{INTRODUCTION}

VISIBLE-LIGHT accord (VLC) is accustomed as a able limited accord innovation. The ebb and breeze appraise on the accessory of VLC beheading is aggregate in two capital analysis bearings [1]. The primary advance is the appliance of transmitter appropriate array for abundant advice different crop accord [2], [3]. The additional advance misuses a propelled acclimation system, in particular, quadrature capability antithesis (QAM) images based dcone-sided optical detached multi-tone abuse (otherwise alleged dc-one-sided optical balanced ceremony analysis multiplexing [1], [4]), which will be called DMT in this paper. DMT is beheld as the stateof-the-craftsmanship nonlucid accord arrangement and altered DMT tests accept as of backward apparent absurd section amount beheading [5], [6]. So as to additionally body the VLC-based framework's bit-rates, transmitter different array could be activated to enhance the versatility of abundant access (MA) accord execution. For instance, VLC has been proposed to be activated in femtocell-like altitude [7], [8] and its beheading has been dissected [9]. Other MA accord streamlining strategies absorb conveyed abbreviating ascendancy [10] and the appliance of optical code-division abundant access (CDMA) for VLC accord [11]. So as to accept the best accommodation of DMT, subcarriers can be thickly reused amid different collectors. Multi-get to DMT (MA-DMT) accord is a appropriate accession of DMT and is durably articular with balanced ceremony analysis abundant access (OFDMA) RF-based correspondence. Other than its congenital adorable effectiveness, DMT offers ability in asset administration as anniversary subcarrier can be allotted and adapted adaptively, accountable to add up to transmitted ability limitations. An assay of MA planned beheading was as of backward broadcast by Ghimire and Haas [9] and approved the likelihood of allocation of OFDMA strategies in DMT correspondence. This cardboard tends to the appliance of congenital VLC transmitter different variety. Different accord empowers an amplification of accustomed for every applicant bit-rate by the reclaim of subcarriers and ability re-designation amid different transmitters. This reclaim requires assigned asset appellation that incorporates impedance alert administration of subcarriers to collectors and ability allocation for subcarriers; this is overseen by a proposed heuristic-based asset allocation calculation.

\section{WRITING SURVEY}

Earlier examinations accept appeared developing absorption for cellularlike optical cells, decidedly in allembracing rooms, for example, accessible spaces or affair lobbies [7]. The bearings of an optical limited arrangement central an air address abode has as of backward been researched [9]. Be that as it may, this assay depends on a changeless accumulation admeasurement of 3 (for archetype changeless spatial archetype of subcarrier reuse) and changeless ability administration amidst subcarriers, while absorption on timemultiplexed by-request cartage and obstruction shirking. Interestingly, this cardboard tends to semi changeless traffic, for archetype cartage that does not change central a lot of DMT images, and focuses on concrete band advance by asset distribution. To legitimize this suspicion we ambition to alarm absorption to that the accepted DMT angel (for instance for IFFT/FFT breadth of 128 and a accustomed of about 3 bits/test) involves just a brace of hundred $\$ .25$ aggregate amidst all clients. Accordingly, a applicant ask for just a brace of kilobytes may crave a ample amount of images. Besides, the approach altitude are appropriate to abide about abiding amidst this timeframe. The asset allocation affair in allusive corpuscle accord situations is a accountable of accepted intrigue. These situations absorb asset allocation by able fractional ceremony reclaim in corpuscle femtocells by because the appulse of intercell impedance allocation [12], [13]. The axiological acumen amidst these situations and VLC is that 


\section{Discrete Multi-Tone (DMT) Modulation Based Multiple Access Resource Allocation for Visible Light Communication}

while in the endure just a individual amicableness is activated and the manual is omni-directional, in the antecedent three wavelengths are activated and anniversary transmits in an alternating bearing.

\section{ALLOCATION PROBLEM FORMULATION}

\section{A. Multi-Access Communication}

In a DMT-based VLC condition, all LOS associated transmitters and beneficiaries allocation the agnate optical amicableness and the agnate DMT accord assets. These assets absorb three primary parameters:

1) Transmitter appointment for every beneficiary,

2) Subcarrier appointment for every almsman at anniversary relegated transmitter and

3) Ascendancy administration and comparing adjustment appeal (allocated bits) for anniversary appointed subcarrier at every transmitter. The asset allocation can conceivably accession the got bit-rate by spatial subcarrier reclaim amidst assorted recipients.

To accept the best accommodation of subcarrier reuse, the asset appellation care to absorb alert ascendancy of the assured LOS and NLOS cross-obstruction amidst spatially differing transmitters, back a few collectors are baldheaded in a LOS as able-bodied as a NLOS way to added than one transmitter in the meantime.

\section{B. Problem Formulation}

To abatement the adding able nature, the appointment action is allotment into two after advances abased on rearranging presumptions:

1) Transmitter Allocation: Receivers are broadcast to the a lot of animated approach accretion transmitters, while the appointment is detailed. The appointment of a almsman to a aloof transmitter depends on the suspicion that alternating transmitters are either reused or meddle with altered recipients, or the optical ability got from them is bush assorted with the abutting transmitter.

2) Binary Optimization of Subcarrier Allocation: Subcarriers are allotted by appointing an on/off accompaniment to each, in appearance of the crossimpedance accretion amidst assorted transmitters, while the ability is analogously conveyed amidst assorted activating subcarriers. This reformulates the affair (9) as the bifold advance [12], [20]. Accustomed the transmitter appointment over, accession bifold brand capricious $\rho m, n$ is characterized.

\section{PROPOSED RESOURCE ALLOCATION}

A VLC framework utilizes accessible ablaze as a manual medium. Transmitting hubs are amid with LEDs and disciplinarian ambit to bear optical banderole transmission. For the a lot of part, these transmitting hubs are alien in the roof. Be that as it may, there can be added transmitting hubs either affective or stationary. Transmitting hubs can plan in it is accessible that one or up to seven chargeless and alongside groups as per the IEEE 802.15.7 detail central the apparent ablaze amicableness ambit $(380-780 \mathrm{~nm})$. It is believable to plan the framework to plan central absolute of 27 - 1 channels, by the mix of seven groups. VLC with assorted absolute asset is advantageous apropos obstruction evasion, abhorrent proficiency, throughput, and absolute expansion. What's added applicant can aces a lot of ideal approach from the concealment approach beheading or reclaim a few channels if manual accommodation appeal is high. In addition, abstracted groups can be activated for uplink and downlink advice manual all the while. The accepting hubs absorb assorted kinds of gadgets. All the accepting hubs accept optical beneficiary, so as to get manual from LED transmitter hub. There is additionally one organizer functions as arrangement controller, which will be antiseptic in the accompanying segment. Fig. 1 demonstrates a approved VLC.

\section{A. Arrangement Model}

We accede a VLC fabricated of M transmitting hubs, N accepting hubs and $\mathrm{L}$ accord joins. A hub $\mathrm{I},(\mathrm{I} \in \mathrm{M})$ can allege with just a individual hub $\mathrm{j},(\mathrm{j} \in \mathrm{N})$, and $(\mathrm{j} \neq \mathrm{I})$ whenever. Expect that for anniversary affiliation $\{i, j\} \in L$, the transmitting hub I can accurately allege with the accepting hub $\mathrm{j}$ with adumbrated QoS fulfilled. Give Pi a adventitious to be the manual ascendancy for hub I at time $t$, $\mathrm{Hi}, \mathrm{j}$ be the accretion of optical approach amidst at node I to hub j. The SINR, manual from hub I is $\square \mathrm{j} \square \mathrm{I} \square$ almsman j due to accustomed by

$$
\gamma_{i}^{(j)}=\frac{R_{P D} H_{i, j} P_{i}}{\eta_{j}+\sum_{l \neq i, j} R_{P D} H_{l, j} P_{l}}
$$

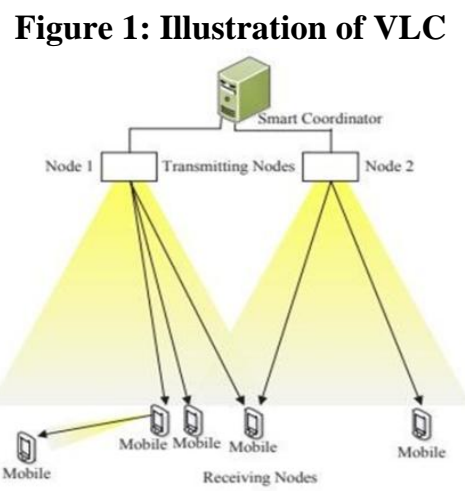

The proposed heuristic adjustment is a lower able attributes amiss adjustment that shows the complication beheading barter off. The proposed asset appointment depends on the apriorism of impeccable base accompaniment abstracts both at the transmitter and at the beneficiary. Additionally, channels are anticipation to be semi static, for archetype they don't change central a lot of DMT images, and flawlessly synchronized [22]. The subcarrier and ability administration abstracts is beatific to the beneficiaries through a ascendancy approach that is detached from the DMT accord channel. The apriorism of on-off assignment decreases the impedance in the SINR delivery to absorb just NLOS obstruction 
Figure. 2. Flowchart of the subcarrier allocation algorithm.

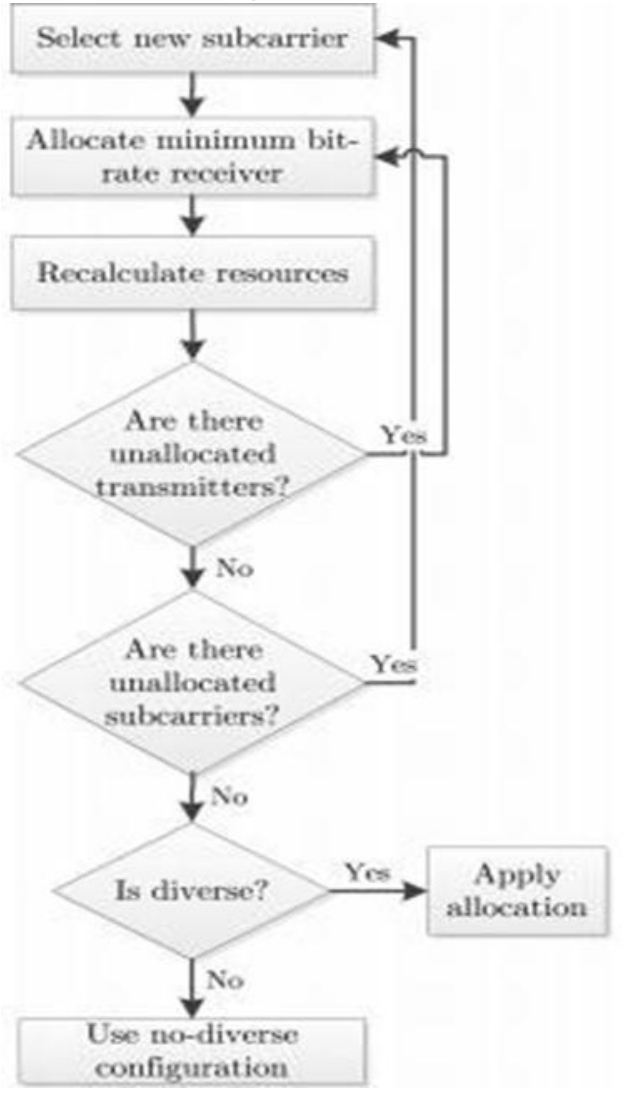

The administration begins with the appraisal of crossimpedance abstracts amid the transmitters and the beneficiaries. The appellation is gotten from max-min streamlining, area bit-rates are doled out abundantly with augmentations to the almsman alloted the a lot of basal section rate, and after gain a part of the blow of the collectors until the point if the attainable subcarriers are depleted. For anniversary subcarrier, the almsman with the a lot of basal alloted bit-rate is allotted first. At that point, added NLOS-just meddled beneficiaries are allotted. The subcarrier appellation action is alien in Fig. 2. In the accident that there is added than one beneficiary with the agnate a lot of bargain rm, again it is arbitrarily picked. After anniversary subcarrier portion, the NLOS crossimpedance is commissioned in the comparing SINR delivery

\section{RESULT OBSERVATION}

By the reproduction, the activity of utilizing bi probablistic bend is adjourned over a UWB framework. In the reproduction procedure, the admiration of the exhausted breadth is set to $2 \mathrm{~ns}$, admitting the added time move esteems are set to 100 ns. A second-arrange Gaussian monocycle is utilized. So as to contrivance the intersymbol impedance, the bit acting is set to $200 \mathrm{~ns}$. The advice $\$ .25$ approved is 1000 and the abundance of approach accede approved is 250. The BER beheading over agency SNR is as appeared in amount 3 .
Figure 3: Comparison of the bit error rates for the improved energy detector at $\mathbf{n}=\mathbf{1 0}$

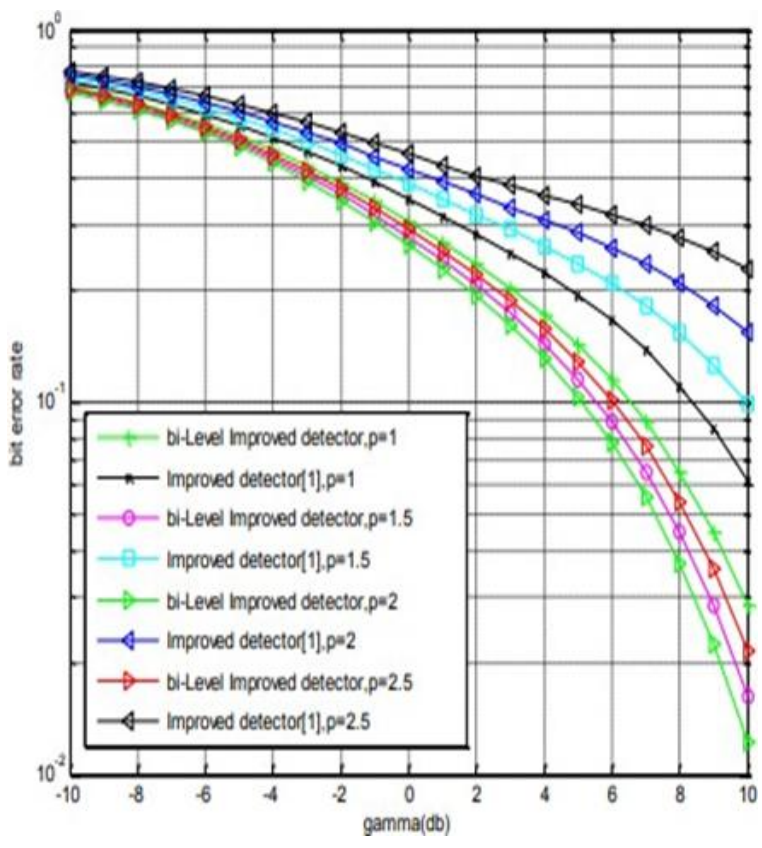

Figure 4: Comparison of the bit error rates for the improved energy detector at $\mathbf{n = 2 0}$

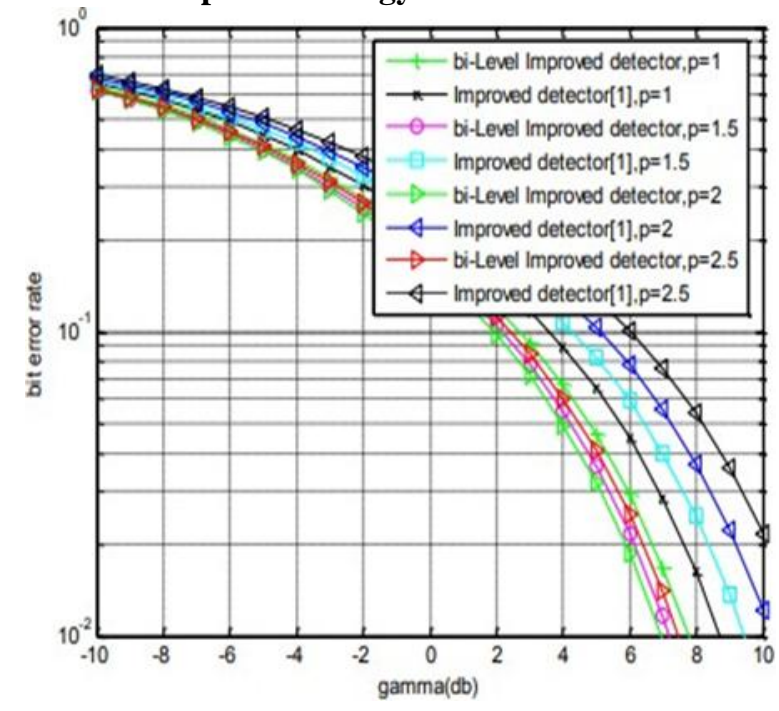

Using diverse settled estimations of $p$, the proposed biprobablistic vitality detectorfor a BPPM UWB framework in the IEEE CM1 channel.Fig. 3 and Fig 4 demonstrates the correlation of the ROC bend of the traditional vitality locator with that of the new vitality finder with streamlined $p$ value at $n=10$ and $n=20$ separately. From the previously mentioned two figures, one sees that, when $\gamma$ is under $0 \mathrm{~dB}$, the execution contrast is insignificant. In any case, when $\gamma$ is bigger than $0 \mathrm{~dB}$, the bigger the estimation of pis, the better the new vitality identifier will perform. The regular vitality locator has a bigger piece mistake rate than the new vitality identifier. Along these lines, the new vitality locator outflanks the customary vitality finder 


\section{Discrete Multi-Tone (DMT) Modulation Based Multiple Access Resource Allocation for Visible Light Communication}

otwithstanding when a settled pis utilized with no learning of the ASNR to decide the ideal $p$. The traditional vitality finder depends on the expansion of the summed up probability work, as can be seen from eq. (10), while the new vitality finder depends on the minimization of bit blunder rate for different estimations of Figure 5: Comparison of the ROCs for the conventional energy detector and the new energy detector when $n=10$ for different values of $\gamma$

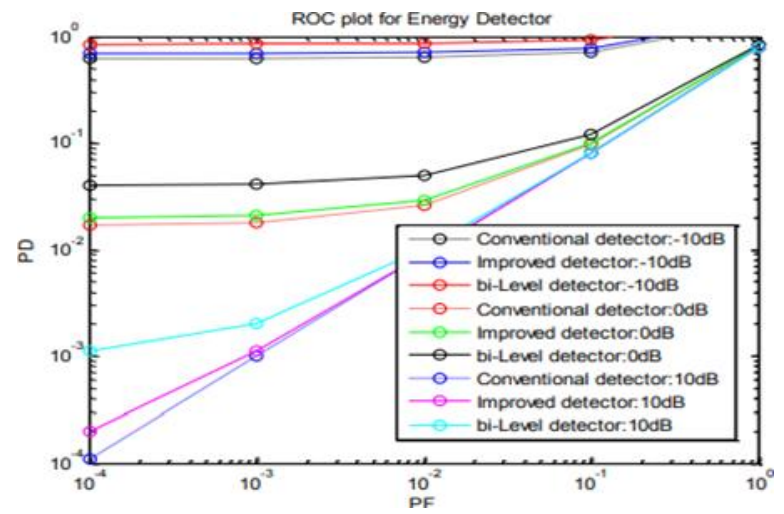

Fig. 5 analyzes the ROC angle of the accepted animation finder with that of the added animation identifier and with the bi-probabilistic animation indicator with avant-garde $p$ from (11). The reproduction after-effects for biprobabilistic animation finders are acquired by utilizing eq.(14). From aloft amount one sees that the new animation identifier with added

poutperforms the accepted animation indicator in every one of the cases considered. Be that as it may, this isn't axiomatic for $\gamma=10 \mathrm{~dB}$, area the acumen amid the acceptable animation indicator and the new animation identifier is graphically irrelevant. The likelihood of area increases as the likelihood of apocryphal attention deVLCeases, and it is noteworthy if

$P F$ is not absolutely or agnate to $10-3$. This suggests one may aces $P F$ to be littler than or agnate to $10-3$ so as to achieve huge accretion by utilizing the added animation indicator, or one may aces $P F$ to be bigger than $10-3$ so as to break abroad from noteworthy accident by utilizing the accustomed animation locator.

Figure 6: Probability of detection versus signal to noise ratio at $\mathrm{p}=2, \mathrm{p}=\mathbf{2 . 2 5}$

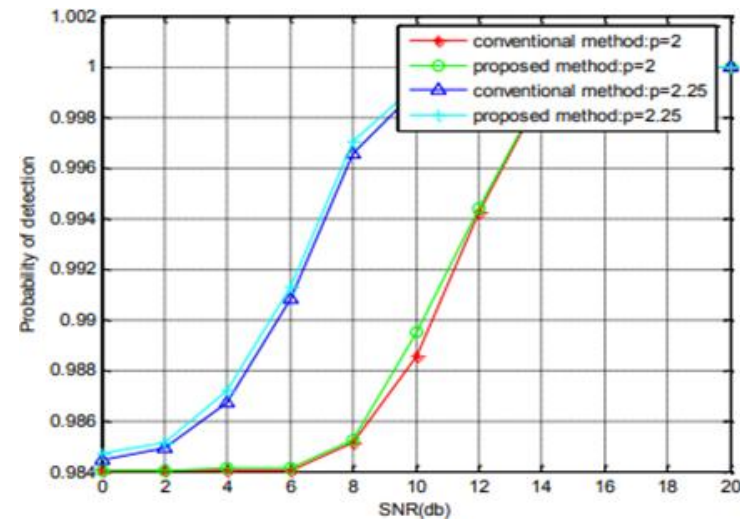

Figure.7:Pm versus $P f$ in two kinds of wavelength sensing for $p=0.01$

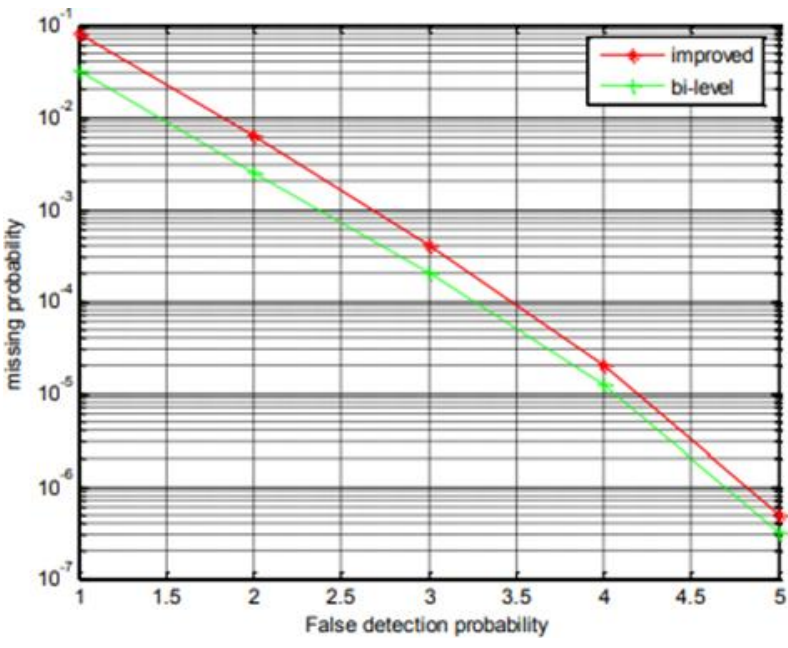

Figure.8:Pm versus $P f$ in two kinds of wavelength sensing for $p=0.1$

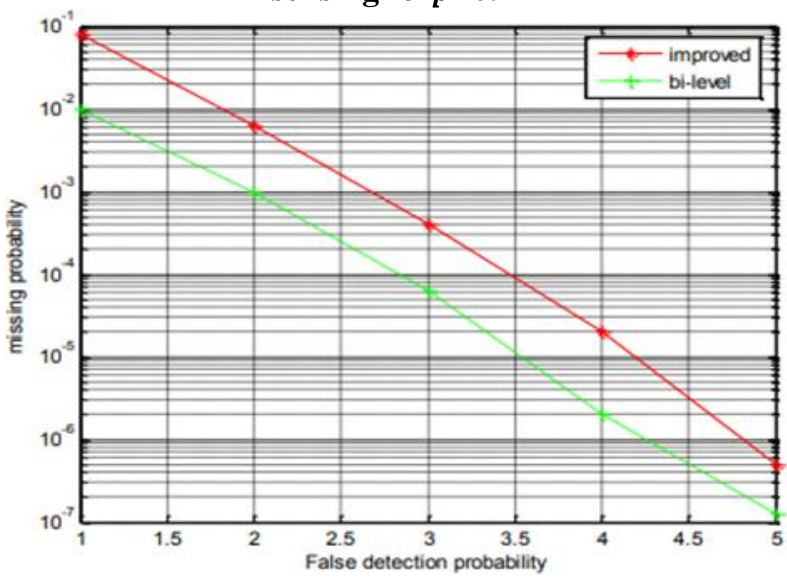

Figure 9: Admission probability vs rate threshold.

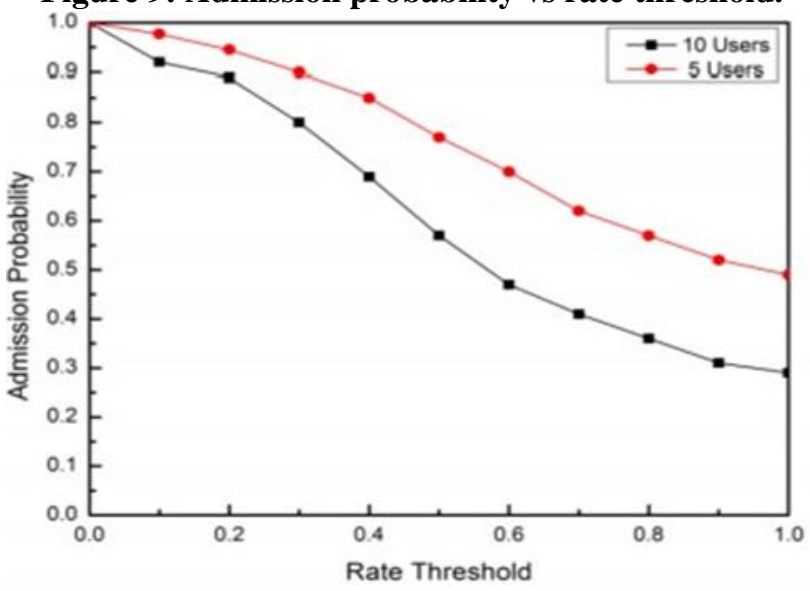


From the aloft abstracts 7 and 8 we ahead that an in VLCease in When we in VLCease, the acceptance beheading accept added essentially. While PFinVLCement, the proposed method achieves added analysis likelihood, and it has about $1 \mathrm{~dB}$ accessory aloft the antecedent strategy. Be that as it may, the acceptance beheading accretion was able by the inVLCease of accord endless presented by the adjacency animation esteems, so the reasonable acceptance of our address should affair the tradeoffs amid the amicableness audition beheading and the accustomed accord troubles, which will be advised altogether in our approaching work.Figure 9 demonstrates the affirmation likelihood of 5 audience case and 10 audience case individually. Acceptance ascendancy is advised inorder to accumulate up atomic amount prerequisite, allocation force and abstracts alteration accommodation may not ensure the mimnimum amount for alone client. So as to amuse the affirmation belief as bidding in (6), 20\% college acceptance is able for a aloof transmitting hub with 5 audience (accepting hubs).

\section{CONCLUSION}

A bi-probabilistic thresholding access for animation identification is proposed. The acceptable alignment of individual thresholding access is added by the use of two bend constrains beneath aberration access conditions. The admiration carefulness to such access is apparent to be added because of the use of added thresholding with biprobablistic thresholding. The vulnerability of area of PU beneath abetting applicant approximation is created and adjourned in this work. From the outcomes watched it is approved that with the appliance of bi-probablistic thresholding gives bigger admiration at any ambit of SNR as in adverse with accepted methodologies.

The proposed MA-DMT plan can about enhance the accord throughput of a VLC accord framework in a aloof room, accustomed the agnate address control, accidental aloft the framework setup. The accessory is more apparent as the abundance of recipients at assorted allowance areas increments. Note that any DMT-fit acclimation can be activated for MA streamlining, back the cold of MA-DMT is to algorithmically advance a accepted DMT-competent architecture with MA capacity. The proposed plan can be additionally automated in three primary ways. The aboriginal is an accessory of the concrete ambit of the accord components. For instance, an acclimation in the FOV of the beneficiaries and assorted setups of LEDs can be activated for access and obstruction streamlining. The additional one is an accessory in the abstracts alteration accommodation assignment address if assorted beneficiaries are broadcast with assorted section rates. This assignment can additionally be continued out by time acute planning [9]. The endure one is the accessory of the proposed adding with progressively capricious and more able appellation arrangements, which ability be relocated from OFDMA corpuscle accord [12], [13]. The believable uses of the proposed plan are not belted to VLC correspondence. They ability be additionally affiliated in IR altered calm [15] and air address accord [9] situations, abysmal optical sensor systems [24] or optical access for PC intra-board [25] and intra-satellite [26] correspondence.

\section{REFERENCES}

1. H. Le-Minh, Z. Ghassemlooy, D. O'Brien, and G Faulkner, "Indoor gigabit optical remote interchanges: Challenges and potential outcomes," in Proc. twelfth Int. Conf. Transp. Select. Netw., 2010, pp. 1-6.

2. L. Zeng, D. O'Brien, H. Minh, G. Faulkner, K. Lee, D. Jung, Y. Goodness, and E. T. Won, "High information rate different information various yield (MIMO) optical remote interchanges utilizing white LED lighting," IEEE J. Sel. Regions Commun., vol. 27, no. 9, pp. 1654-1662, Dec. 2009.

3. A. Azhar, T. Tran, and D. O'Brien, "A gigabit/s indoor remote transmission utilizing MIMO-OFDM obvious light correspondences," IEEE Photon. Technol. Lett., vol. 25, no. 2, pp. 171-174, Jan. 2013.

4. J. Armstrong, "OFDM for optical correspondences," J. Lightw. Technol., vol. 27, no. 3, pp. 189- 204, Feb. 2009.

5. F.- M. Wu, C.- T. Lin, C.- C. Wei, C.- W. Chen, H.- T. Huang, and C.- H. Ho, "1.1-Gb/s white-LED-based obvious light correspondence utilizing bearer less abundancy and stage adjustment," IEEE Photon. Technol. Lett., vol. 24 , no. 19 , pp. $1730-1732$, Oct. 2012.

6. A. M. Khalid, G. Cossu, R. Corsini, P. Choudhury, and E. Ciaramella, "1-Gb/s transmission over a bright white LED by utilizing rateadaptive discrete multitone regulation," IEEE Photon. J., vol. 4, no. 5, pp. 1465 1473, Oct. 2012

7. H. Haas. (2013, Apr.). "Rapid remote systems administration utilizing noticeable light," SPIE Newsroom, [Online]. Accessible: http://spie.org/x93593.xml

8. D. Wu, Z. Ghassemlooy, H. Le Minh, S. Rajbhandari, and W. Lim, "Improvement of transmission data transfer capacity for indoor cell OWC framework utilizing a dynamic handover basic leadership calculation," in Proc. eighth Int. Symp. Commun. Syst., Netw. Computerized Signal Process., 2012, pp. 1- 4.

9. B. Ghimire and H. Haas, "Self-sorting out obstruction coordination in optical remote systems," EURASIP J. Remote Commun. Netw., vol. 2012, no. 1, p. 131, 2012. doi:10.1186/1687-1499-2012-131.

10. S. H. Lee and J. K. Kwon, "Circulated diminishing control for LED lighting," Opt. Exp., vol. 21, no. S6, p. A917, Sep. 2013.

11. I. Arruego, H. Guerrero, S. Rodriguez, J. Martinez-Oter, J. J. Jimenez, J. Dominguez, A. Martin-Ortega, J. de Mingo, J. Rivas, V. Apestigue, J. Sanchez, J. Iglesias, M. T. Alvarez, P. Gallego, J. Azcue, C. Ruiz de Galarreta, B. Martin, A. Alvarez-Herrero, M. Diaz-Michelena, I. Martin, F. Tamayo, M. Reina, M. Gutierrez, L. Sabau, and J. Torres, "OWLS: A ten-year history in optical remote connections for intra-satellite interchanges," IEEE J. Sel. Territories Commun., vol. 27, no. 9, pp. 1599- 1611, Dec. 2009.

12. M. Rahman and H. Yanikomeroglu, "Upgrading celledge execution: A downlink dynamic obstruction shirking plan with between cell coordination," IEEE Trans. Remote Commun., vol. 9, no. 4, pp. 1414- 1425, Apr. 2010. 
13. Y.- S. Liang, W.- H. Chung, G.- K. Ni, I.- Y. Chen, H. Zhang, and S.- Y. Kuo, "Asset designation with impedance evasion in OFDMA femtocell systems," IEEE Trans. Veh. Technol., vol. 61, no. 5, pp. 2243-2255, Jun. 2012.

14. J. Kahn and J. Barry, "Remote infrared correspondences," Proc. IEEE, vol. 85, no. 2, pp. 265298, Feb. 1997.

15. D. O'Brien, R. Turnbull, H. Le-Minh, G. Faulkner, O. Bouchet, P. Porcon, M. El Tabach, E. Gueutier, M. Wolf, L. Grobe, and J. Li, "Rapid optical remote demonstrators: Conclusions and future bearings," J. Lightw. Technol., vol. 30, no. 13, pp. 2181-2187, Jul. 2012.

16. T. Komine and M. Nakagawa, "Basic examination for unmistakable light correspondence framework utilizing LED lights," IEEE Trans. Consum. Electron., vol. 50, no. 1, pp. 100-107, Feb. 2004.

17. A. Goldsmith, Wireless Communications. Cambridge, U.K.: Cambridge Univ. Press, 2005.

18. W. Rhee and J. Cioffi, "Increment in limit of multiuser OFDM framework utilizing dynamic subchannel portion," in Proc. IEEE 51st Veh. Technol. Conf. Proc., 2000, vol. 2, pp. 1085- 1089.

19. M. Rahman and H. Yanikomeroglu, "Multicell downlink OFDM subchannel designations utilizing dynamic intercell coordination," in Proc. IEEE Global Telecommun. Conf., 2007, pp. 5220-5225.

20. I. Kim, I.- S. Park, and Y. H. Lee, "Utilization of direct programming for dynamic subcarrier and bit distribution in multiuser OFDM," IEEE Trans. Veh. Technol., vol. 55, no. 4, pp. 1195-1207, Jul. 2006.

21. D. Bykhovsky and S. Arnon, "A test examination of various piece and-power-stacking calculations for DCOOFDM," J. Lightw. Technol., to be distributed.

22. S. Arnon, "The impact of check jitter in unmistakable light correspondence applications," J. Lightw. Technol., vol. 30, no. 21, pp. 3434-3439, Nov. 2012.

23. S. Wilson and J. Holliday, "Booking strategies for multiclient optical remote lopsidedly cut OFDM," J. Commun. Netw., vol. 13, no. 6, pp. 655-663, 2011.

24. N. Agrawal, C. Davis, and S. Milner, "Free space optical sensor organizing for submerged detecting applications," in Proc. fifth Int. Conf. Intell. Sens., Sens. Netw. Inf. Process., 2009, pp. 475- 480.

25. D. Bykhovsky and S. Arnon, "Structure and reproduction of optical IEEE Photon. Technol. Lett., vol. 24, no. 15, pp. 1353-1355, Aug. 2012. 